INPLASY

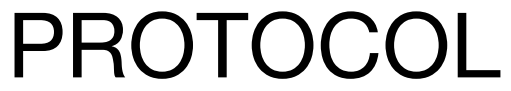

To cite: Yang et al.

Effectiveness and safety of transcutaneous electrical nerve stimulation (TENS) for pain relief in labour: a protocol for systematic review and metaanalysis. Inplasy protocol 202070018. doi: 10.37766/inplasy2020.7.0018

Received: 05 July 2020

Published: 05 July 2020

Corresponding author: Jiao Yang

yangjiao@stu.cdutcm.edu.cn

Author Affiliation:

Chengdu University of

Traditional Chinese Medicine

Support: None.

Review Stage at time of this submission: The review has not yet started.

Conflicts of interest:

The authors declare that they have no competing interests.

\section{Effectiveness and safety of transcutaneous electrical nerve stimulation (TENS) for pain relief in labour: a protocol for systematic review and meta-analysis}

Yang, J1; Zhao, S2; Xu, G3; Liang, F4; Zhao, L5.

Review question / Objective: Is transcutaneous electrical nerve stimulation (TENS) an effective way for pain relieve in labour? Is transcutaneous electrical nerve stimulation (TENS) a safe therapy for pain relieve in the mother and the fetus? Are women satisfied with pain relief during labour?

Condition being studied: Pain during delivery is a complex, physiological, subjective and multidimensional response to the sensory stimuli principally generated by uterine contraction. Pain control plays an important role during labor because it contributes to the physical well-being of both mother and fetus. TENS has been widely used for pain in labour. But, there is still controversial of its effectiveness and safety. Hence, we planned to perform a systematic review to evaluate the effectiveness and safety of TENS for pain in labour.

INPLASY registration number: This protocol was registered with the International Platform of Registered Systematic Review and Meta-Analysis Protocols (INPLASY) on 05 July 2020 and was last updated on 05 July 2020 (registration number INPLASY202070018).

\section{INTRODUCTION}

Review question / Objective: Is transcutaneous electrical nerve stimulation (TENS) an effective way for pain relieve in labour? Is transcutaneous electrical nerve stimulation (TENS) a safe therapy for pain relieve in the mother and the fetus? Are women satisfied with pain relief during labour?

Condition being studied: Pain during delivery is a complex, physiological, subjective and multidimensional response 
to the sensory stimuli principally generated by uterine contraction. Pain control plays an important role during labor because it contributes to the physical well-being of both mother and fetus. TENS has been widely used for pain in labour. But, there is still controversial of its effectiveness and safety. Hence, we planned to perform a systematic review to evaluate the effectiveness and safety of TENS for pain in labour.

\section{METHODS}

Search strategy: The following databases will be searched from their inception to Dec 2019: Cochrane Library, MEDLINE, EMBASE, Ovid, Chinese National Knowledge Infrastructure (CNKI), Wanfang Database, the Chongqing VIP Chinese Science and Technology Periodical Database (VIP), Chinese Biomedical Literature Database (CBM), World Health Organization Clinical Trials Registry, ClinicalTrials.gov, and reference lists of articles to identify additional studies, The following medical search headings (MeSH) will be used: "TENS", "Transcutaneous Electrical Nerve Stimulation", "Stimulation Transcutaneous", "labor", "labour", "labor pain", "labour pain", "labor, obstetric", "labour, obstetric", "analgesia, obstetric", "childbirth" and "parturition". "randomized controlled trial"," randomised controlled", "randomised, controlled", "clinical trial". Chinese translations of these search terms will be used for the Chinese databases.

Participant or population: This study will include Women in labour. The inclusion criteria were as follows: (1) Aged above 18; (2) A gestational age between 37 and 42 weeks; (3) Had cervical dilatation of 3-5 cm; (4) Demonstrated the ability to have verbal communication and agreed to participate in the study. Exclusion criteria included the following: (1) aged below 18; (2) cutaneous damage at the TENS application sites; (3)women wearing a pacemaker or automatic implanted cardiac defibrillator; (4) inability to understand or refusal to sign the informed consent form.
Intervention: The patients who accept TENS for labour pain would be included by experimental group; the types of TENS equipment and the applicated location will not be restricted. TENS used alone or as add-on other therapy will be included in this study.

Comparator: The following control group will be considered: 1 . placebo TENS device 2. routine care 3 . pharmacological interventions 4. other non-pharmacological interventions Studies that only compare different doses of TENS for pain shall be excluded.

Study designs to be included: The review will include randomised controlled trials (RCT) that were reported in any language. Trials using a two-arm or three-arm parallel design wil.

Eligibility criteria: Studies met the following inclusion criteria were included: (i) patients should be women in labour with gestational age between 37 and 42 weeks; (ii) the patients who accept TENS for labour pain would be included by experimental group; (iii) randomized controlled trials (RCT) on human; (iv) the control group would be placebo ENS device, routine care, pharmacological interventions or other non-pharmacological interventions; (v) the primary outcome was the pain intensity in labour and satisfaction with pain relief during labour. Accordingly, case reports, case series, editorials, in vitro experiments and animal studies were excluded. If multiple studies reported overlapping data, the most comprehensive one was included in the meta-analysis. If only abstract was available, the corresponding author of the abstract would be contacted for raw data via email. The record would be discarded if the author can't be contacted after we sent the email for at least three times.

Information sources: If multiple studies reported overlapping data, the most comprehensive one was included in the meta-analysis. If only abstract was available, the corresponding author of the abstract would be contacted for raw data via email. The record would be discarded if 
the author can't be contacted after we sent the email for at least three times.

Main outcome(s): Pain intensity in labour and Satisfaction with pain relief during labour.

Additional outcome(s): Outcomes for Maternal 1. duration of labour, 2. cervical dilation on admission to hospital, 3.augmentation of labour, 4.use of other methods of pain relief during labour 5 . assisted birth or caesarean section, 6.side effects, 7. sense of control in labour ; 8.satisfaction with childbirth experience. 9.postpartum bleeding outcome for Fetal/ neonate 1.Apgar score less than seven at five minutes 2.Cord blood pH less than 7.1 3.Adverse events.

Data management: Two reviewers will preliminary filter the article by screening the titles, abstracts, and keywords. Duplicated and ineligible mismatched research will be removed. Then, we will further evaluate the studies by reading the full text. Excluded studies will be recorded with reasons as an Excel data set. Furthermore, we will independently extract data from the selected studies, and any disagreement will be resolved through discussions or negotiation with a senior reviewer (Qian Hua Zheng). The following information will be extracted: title, year of publication, first author's name and affiliation, country, sponsor, sample size, details of participants, intervention details, type of control, results (outcome measures, adverse events) and conclusion. If necessary we will contact the first or corresponding authors via e-mail for additional information.

Quality assessment / Risk of bias analysis: The risk of bias for each selected trial will be performed by two independent reviewers (Qing-Yu Yi and Ying Cheng) using the Cochrane Collaboration's tool. Disagreements will be resolved by discussion or consulting the third reviewer (Ling Zhao). We used the following six separate criteria: (1) random sequence generation,; (2) Allocation concealment; (3) Blinding (performance bias and detection bias); (4) Incomplete outcome data; (5) Selective reporting; (6) Other bias. Particularly, we will use the Grades Profiler as the Grading of Recommendation, Assessment, Development, and Evaluation (GRADE) system to grading the quality of the evidence.

Strategy of data synthesis: We will use the Review Manager software V.5.3 to carry out statistical analysis. If there is no statistical heterogeneity among the results, a fixedeffects model will be used for metaanalysis. Otherwise, the heterogeneity source will be further analyzed and a random-effects model will be used for meta-analysis after excluding the effects of significant clinical heterogeneity. But when there is significant clinical heterogeneity, we will use subgroup analysis or sensitivity analysis, or only descriptive analysis.

Subgroup analysis: If one of the outcome parameters demonstrates statistically significant differences between intervention groups, we will plan to use subgroup analyses. Planned subgroup analyses will be performed in: different types of TENS, different application sites of TENS, parity (nulliparous versus multiparous women),stage of labour (first stage latent versus active phase), spontaneous labour versus induced labour, term versus preterm birth, continuous support in labour versus no continuous support.

Sensibility analysis: In order to evaluate the robustness and validity of the results, the leave-one-out sensitivity analysis will be conducted by removing each study in turn and reevaluating the resulting effect on the overall estimate.

Country(ies) involved: Chinam, Switzerland.

Keywords: transcutaneous electrical nerve stimulation (TENS), labour, meta-analysis.

Contributions of each author:

Author 1 - Jiao Yang - Author 1 designed the research and will draft the manuscript. 
Author 2 - Sha Zhao - Author 2 will participate in the acquisition of data and perform the meta-analyses.

Author 3 - Guixing Xu - Author 3 will participate in the acquisition of data and perform the meta-analyses.

Author 4 - Fanrong Liang - Author 4 will revise the manuscript.

Author 5 - Ling Zhao - Author 5 will revise the manuscript. 